\title{
Linx
}

Revue des linguistes de l'université Paris X Nanterre

$53 \mid 2005$

Le semi-figement

\section{Présentation. Le semi-figement}

\section{Antoinette Balibar-Mrabti et Céline Vaguer}

\section{OpenEdition \\ Journals}

Édition électronique

URL : http://journals.openedition.org/linx/252

DOI : 10.4000/linx.252

ISSN : 2118-9692

\section{Éditeur}

Presses universitaires de Paris Nanterre

\section{Édition imprimée}

Date de publication : 1 décembre 2005

Pagination : 7-15

ISSN : 0246-8743

\section{Référence électronique}

Antoinette Balibar-Mrabti et Céline Vaguer, « Présentation. Le semi-figement », Linx [En ligne],

53 | 2005, mis en ligne le 09 février 2011, consulté le 21 avril 2019. URL : http://

journals.openedition.org/linx/252 


\title{
Présentation. Le semi-figement
}

\author{
Antoinette Balibar-Mrabti et Céline Vaguer \\ Université Paris $X$ - Nanterre et Laboratoire MoDyCo (CNRS \\ $U M R$ 7114)
}

\section{Semi-figement, figement, degrés de figement}

L'origine du numéro 53/2005 de Linx est une journée internationale d'études sur le thème du semi-figement, organisée le 25 mars 2005 par Antoinette Balibar-Mrabti et Céline Vaguer dans le cadre de l'Opération «Syntaxe et sémantique descriptives du français » dirigée par Danielle Leeman au sein du laboratoire MoDyCo et soutenue par l'Ecole doctorale "Connaissance, langage, modélisation» de l'université de Paris XNanterre. Pourquoi avoir centré nos confrontations de méthodes et de résultats sur le semi-figement, un terme suggestif mais sans statut théorique? De fait, les quatorze articles de ce numéro, dès qu'on se reporte aux intitulés retenus par les auteurs, mettent immédiatement en évidence ce que le terme en lui-même évoque déjà. Il fait son apparition aux franges de la notion beaucoup mieux définie et admise de figement.

Pour faire saisir immédiatement en quoi notre choix thématique peut être utile, rappelons le statut de la question du figement et son évolution dans la théorie et dans la description linguistique. En première étape, la syntaxe est avec Saussure le domaine de la parole et du discours où se place la liberté de l'individu dans les contraintes générales du système. De ce point de vue, il y a marginalisation des locutions et expressions. Elles présentent, certes, des traces diachroniques mais en synchronie le procédé mis en œuvre s'est bloqué en s'arrêtant à des variantes sémantiquement imprévisibles. Renvoyées à l'expressivité, à la figure, elles relèvent des formes stylistiquement enrichies. En deuxième étape, autour de la grammaire transformationnelle et 
générative, la syntaxe n'est plus le seul domaine de l'individu donc de la liberté. Les expressions idiomatiques, et d'une façon générale le figement, s'étudient avec les phrases libres. En syntaxe française deux articles de fond ont symbolisé ce tournant pris par les recherches linguistiques, à partir des travaux de Harris et Chomsky : Une classification des phrases "figées" du français (M. Gross 1982); Du bon usage des expressions idiomatiques dans l'argumentation en syntaxe générative (N. Ruwet 1983). En troisième étape, les expressions viennent sur le devant de la scène avec d'autant plus de poids que les outils informatiques permettent d'optimiser leurs procédures de reconnaissance dans les énoncés ainsi que leurs consultations dans des classements volumineux de type « lexiques-grammaires ». Les travaux nombreux et diversifiés conduisent à s'interroger avec une précision croissante sur la façon dont les facteurs de blocage pèsent sur les facteurs de liberté (et inversement) dans les productions langagières. Fondamentalement, la langue serait à considérer comme le lieu du figé, le libre apparaissant comme relativement marginal avec un continuum, et non plus du discret, du plus figé au libre, en passant par les collocations et le semi-figé.

Mais que se passe-t-il exactement dans cet entre-deux des traitements que par commodité et par raccourci nous nommons «semi-figement»? En vingt-cinq ans de travaux, la question a bien souvent été posée, une des réponses données parmi les plus précises étant l'étude des degrés de figement. Toujours active, elle pourrait requérir plus que jamais notre attention et ouvrir vers une quatrième étape, celle qu'il nous intéresse d'anticiper et de mesurer ici dans ce numéro 53 de la revue Linx. En effet, si les recherches qui ont donné droit de cité au figement en syntaxe post-saussurienne soutiennent aussi durablement l'intérêt, c'est qu'elles se prêtent à des réexamens qui demeurent cruciaux en méthodologie comme en théorie. Un des fils conducteurs argumenté ici est qu'elles demanderaient à être à leur tour pondérées dans le sens d'un rééquilibrage en faveur de facteurs de liberté, certes, tempérés en degrés, mais bel et bien omniprésents à de rares exceptions près. Cette liberté n'aurait-elle pas été minorée dans la théorie par un point de vue sur le figement devenu inflationniste? Une pluralité d'interrogations, avec leurs esquisses de solutions, amène ainsi à valoriser des problématiques, riches des observations que le semi-figement leur fournit. Dans le flou que celui-ci ne manque pas d'introduire, on peut voir un avantage. Nous disposons là d'un espace qui est laissé d'entrée de jeu à notre appréciation. Pour nous résumer, le semi-figement mérite notre attention parce qu'il s'inscrit dans un mouvement naturel de retour critique des syntacticiens sur leurs résultats.

\section{Syntaxe et phrase}

Soulignons en présentation trois courants interdépendants qui, en faisant pression sur la syntaxe, changent notablement nos rapports, intuitifs ou raisonnés, à son objet premier de description, la phrase, donc les «cadrages » syntaxiques du semifigement. C'est d'abord le « retour de la morphologie » et un rapport lexique/texte qui fait l'économie de passer par la syntaxe comme discipline contemporaine autonome. C'est aussi le poids de l'informatique et des aides documentaires qui précisément facilitent les «retours aux corpus textuels». C'est l'importance grandissante des supports audiovisuels modernes. Ils apportent de nouvelles exigences pour l'étude 
formelle de l'expression, ils relativisent la phrase (canonique) adaptée au code écrit, ils confortent l'importance de la phonologie. Dans cette progression des énoncés oraux « directement» enregistrés, sources de documents de mieux en mieux consultables et de plus en plus fiables, de quel réel se nourrit exactement l'observation ? Que veut-on décrire et formaliser dans les modèles?

\subsection{Outils de transcription et texte oral}

Avec les redistributions complexes du rapport oral/écrit, des questions de procédure qui appartenaient à la linguistique dite «de terrain» redeviennent fondamentales. Que transcrire et avec quels outils de transcription ? Utiliser les «moyens orthographiques » à partir de nos habitudes du code écrit reste une commodité pour «lire de l'oral». Cependant dans les interfaces entre langages de programmation et langues naturelles, où l'éventail des conventions et des notations adaptées aux formalismes de l'informatique linguistique se complexifie et se spécialise, ce sont des besoins techniques propres qui sont appelés à se développer sur les transcriptions phonétiques usuelles. Du côté de l'écrit, ce qui reste inchangé, c'est bien sûr l'approche " classique » des différences de registres et de niveaux de langue quand elles passent par le travail du style - et de l'esthétique - avec la littérature (théâtre, roman, nouvelle, récit de vie, etc.) et les fonctions langagières au cinéma (dialogue de scénario, soustitrage, voix off, etc.). Nous y observons le français "populaire ", "familier», 《argotique », que Françoise Gadet a proposé d'appeler « ordinaire » quand l'étude de l'oral et de l'écrit se veut neutre. Ce deuxième type d'accès «traditionnel » à l'oral est incontournable dans toute documentation de linguistique diachronique. Dans les approches synchroniques, ce serait donc plutôt l'inverse. Restituer par écrit les énoncés oraux est de moins en moins convaincant puisque la procédure a beaucoup perdu de son utilité première. Nous pouvons nous en passer pour fixer les «faits de langue parlée» et les faire exister comme objets de science «observables». L'outil orthographique devient encore plus indirect avec des fonctions plus «culturelles", plus souterraines. Envisager pour autant qu'il puisse disparaitre à court terme de la description linguistique de l'oralité serait caricatural. C'est tout le statut historique et mental de l'orthographe qui est précisément en question.

Un faisceau de critères exigerait surtout des efforts accrus pour donner des définitions appropriées et claires de la notion, devenue centrale, de texte oral. On sait qu'elle s'est problématisée dans les «folklores» dont la transmission a d'abord été pensée en marge de l'écriture. Avec les supports audio-visuels contemporains, des partages de fonction entre l'oral et l'écrit se redessinent. Les nouvelles associations qui mettent en jeu le son, l'image et les signes graphiques ont, sur l'essentiel, des propriétés qu'on attend d'un texte : elles s'archivent, elles ont une valeur documentaire. De ce point de vue, elles préfigurent des réajustements de normes et de styles, où l'oral, s'il tend à primer sur l'écrit, ne l'exclut pas pour autant. Dans le champ socio-historique de la latinité, les travaux récents apportent des évaluations différentielles précieuses pour tenter de cerner ce type de modernité encore diffus.

Toute une terminologie du latin classique, en mettant en avant la dimension de l'oralité, nous fait réfléchir sur les paradoxes, anciens et contemporains, de l'acte d'écrire. A l'époque de Cicéron, on connait les œuvres par la « récitation », la « lecture 
publique ». Parmi les techniques enseignées pour maittriser l'art d'écrire et de penser que la rhétorique a codifiées, on apprend à composer avec des exercices de "dictio ", d'« elocutio », de "praelectio ». D'où le verbe latin « dictare », employé pour désigner l'acte de rédiger, quand il faut créer le vieil haut allemand écrit, dans l'Europe du 9ème siècle. Les glissements d'interprétation (qui peuvent aller jusqu'au contresens hors contexte) lorsqu'on emploie en français moderne des termes équivalents comme « diction», « dicter », «élocution» (" prélection» a disparu), mettent en évidence la nécessité de périodiser les fonctions de l'«écriture» dans la communication et la variation linguistiques dont notre actualité propre est un aboutissement. Qu'est-ce qui contribue à reconduire une grammaire et une syntaxe modernes ? Comment la langue écrite et ses techniques, prises dans les limites strictes du code graphique, vont-elles s'adapter aux changements de supports? Vont-elles survivre comme voie royale de l'inscription? Bien comprendre cette complexité, ses contradictions et leurs résolutions, est devenu une des questions les plus intéressantes de la recherche linguistique. C'est une des raisons de l'intérêt porté à la dyslexie en cognition.

\subsection{Morphologie et données d'observation : la citation, la phraséologie}

Avec des modèles comme les "grammaires lexicalisées", la syntaxe s'est durablement investie dans le lexique. Il devient son talon d'Achille. Considérons le cas suivant. Il est des plus révélateurs. Pour raffiner les relations sens/texte, on peut décider de partir de la morphologie, choisie comme base formelle pour définir le lexique. C'est alors un type d'éviction de la syntaxe qui s'enclenche et cela avec d'autant plus de facilité qu'on rejoint des méthodologies "éprouvées» de la linguistique, soutenues par le poids avéré des habitudes en philologie et en critique littéraire. Sur des corpus écrits et oraux de plus en plus volumineux, une cascade de priorités à "redécouvrir » s'installe. Vue comme un artefact de l'analyse en syntaxe, la phrase devient un objet désuet si la morphologie s'en passe. Dès lors, les données d'observation participent d'une vision " rénovée » des enchaînements textuels dans une relation directe lexique/texte. On décentre la syntaxe à l'intérieur d'une morphosyntaxe élargie aux macrosyntaxes, on raffine la morphophonologie et son rapport avec la phonétique. Ces approches remettent au centre des descriptions les dérivations mais aussi les flexions, ce qui n'est pas sans rappeler le rôle historique joué par la grammaire latine dans la constitution des métalangages grammaticaux pour les langues vivantes: la « rection », avec la notion de «cas régime », précédant les notions de «fonction » et de « complément ».

Dans ses effets les plus radicaux, la morphologie éloigne la phrase de deux possibilités habituelles d'isomorphie, l'adéquation phrase de base/prédication et l'adéquation phrase complexe/période. Elle la coupe de la syntaxe et de ses arrièreplans logiques ou rhétoriques, elle la renvoie à sa nature pré-théorique : une portion d'énoncé offrant un cadrage intuitif aux unités du lexique à observer en emploi. La phrase reprend alors un sens qu'on pourrait qualifier d'étymologique. Elle retrouve une parenté avec la citation dont il faut réexaminer toutes les facettes (la récitation en est une). Instrument didactique et mode de culture, la citation coïncide avec l'exemple de grammaire et de dictionnaire mais aussi avec le travail, multiséculaire, des compilations et des phraséologies que nous pratiquons plus que jamais depuis que 
l'informatique documentaire les a modernisées. Or le terme de phraséologie est précisément couramment adopté pour l'étude des degrés de figement chez les linguistes, russophones, hispanophones, germanophones. En syntaxe comme en lexicologie, ce choix terminologique demeure crucial. Ne serait-ce qu'implicitement, lorsqu'on évacue la diachronie, il témoigne de rapprochements incontournables, car liés à la notion d'«usage» dans son rapport avec la norme, qui concernent les conditions de formation du lexique. A travers l'évolution de ses contenus de définition, sa persistance nous rappelle que l'étude du figement va de pair avec une meilleure compréhension des mécanismes de l'acquisition et de la pragmatique pensés en termes de «longues durées ». Il y a là un nœud de disciplines que nous retrouverons souligné par bon nombre des articles que nous présentons.

\subsection{Lexique et informatique : dictionnaires électroniques, lemmatisation}

Assurément les révisions du statut de la syntaxe sont un paradoxe puisque nous

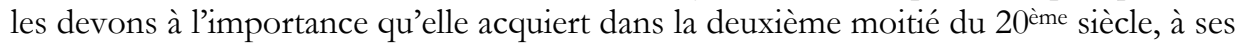
exigences de rigueur souvent jugées exemplaires en sciences sociales durant cette période. C'est donc l'envers d'un succès qu'il s'agit ici de mieux comprendre. Comment en prendre la mesure exacte? Cette question continue de captiver tout chercheur en sciences du langage par l'importance de ses enjeux théoriques et appliqués. Ce qui est sûr, c'est qu'il faut rendre de plus en plus opératoires les outils de la linguistique informatique devenus indispensables pour explorer et traiter les données en corpus. Ce qui est acquis et devient un objectif prioritaire, c'est l'idée d'organiser le lexique, en situation de plaque tournante des propriétés de forme et de sens.

Un des résultats les plus importants nous vient des dictionnaires électroniques, initiés par Maurice Gross, Jean Dubois, Igor Mel'čuk, pour le français. Des propriétés syntaxiques comme la valence sont une base de classement pour les emplois verbaux. Les données, traitées à l'échelle des lexicographes, sont exploitables informatiquement. La linguistique appliquée pèse de tout son poids sur la linguistique générale. Ces dictionnaires d'un type nouveau ont anticipé dès les années 1980 sur les besoins contemporains en aides documentaires, en aides à la traduction et à la rédaction, en didacticiels. Le regain d'intérêt pour la morphologie flexionnelle peut être vu comme une conséquence directe des modélisations informatiques autour des procédures de lemmatisation. Sans ces procédures, l'accès automatique aux entrées lexicales d'un dictionnaire comme aux mots d'un texte numérisé perd une part majeure de son efficacité.

\subsection{La lecture comme nouvean standard: phonologie et grammaire de construction}

L'audio-visuel a renvoyé l'écrit à un statut antérieur à la scolarisation généralisée et placé les spécialistes devant l'urgence de repenser le rapport phonie/graphie. «Défense de déposer de la musique le long de mes vers! » avait pu dire Victor Hugo. Un propos étrangement daté pour nous. Symptomatique d'une survalorisation du français comme code écrit, allant jusqu'à dominer les modèles d'expression poétique, il valorise paradoxalement la «musicalité» de la langue. Bien circonscrite par les 
historiens, cette période, qui culmine en France entre 1850 et 1950, donne beaucoup à réfléchir car elle nous fait mesurer combien la rupture de mentalités est devenue profonde cinquante ans plus tard. L'autonomie de l'écrit a perdu toute évidence et avec elle une bonne partie de son prestige, celui d'être le véhicule par excellence de la communication pour chacun et pour tous.

L'abandon du culte de l'orthographe dans l'enseignement du français est révélateur. Il a glissé par étapes vers de simples objectifs d'hypercorrection. C'est la lecture qui reste au centre du dispositif de socialisation. Ce nouvel ancrage éducatif va de pair avec une discipline comme l'orthophonie (devenue essentielle dans les accompagnements scolaires). En amont des applications pédagogiques et techniques, les approches de type grammaire de construction en phonologie illustrent bien comment se précisent et se choisissent les normes requises pour les standards de prononciation (avec leurs marges de variation) dynamisées par nos exigences de parité avec les normes graphiques (et leurs variations).

\section{L'objet-langue : l'argument d'autorité, le recours aux concordanciers}

Depuis que la conception romantique de l'écrivain, garant du génie de la langue, n'a plus cours en sciences du langage - c'est un des aspects de notre modernité qui rompt avec les prestiges de l'écrit dans la période antérieure - comment se reconduit, se réorganise, disparaît ou se met seulement en retrait, l'argument d'autorité qui sert à trancher entre les usages? Qu'est-ce qui différencie en linguistique française un exemple de grammaire appuyé par une citation d'auteur dans le Littré ou le Trésor de la Langue Française d'une concordance fournie par les aides documentaires contemporaines à partir de la Presse que ne valorisera plus aucune signature particulière dans un Robert \& Collins bilingue récent ou dans le Lexis? C'est précisément là qu'un tour d'horizon, même lacunaire, sur le semi-figement, peut s'avérer particulièrement stimulant car il nous met dans l'obligation de cibler des espaces de variation et de faire le tri - en connaissance de cause - entre deux grandes catégories de transgressions : celles qui sont jugées "naturelles», parce qu'elles sont "systémiques », et celles qui sont à considérer comme étant «hors jeu», les «fautes » (barbarismes et solécismes dans les exercices traditionnels de thème) dont la grammaire dite « descriptive » s'est affranchie.

S'il est vrai que nous avons disqualifié les grands écrivains pour représenter, en termes «stylistiques » et « historiques », des types d'écarts (en synchronie) et des types de différences (en diachronie), tous jugés internes à la langue considérée, où se placent désormais les démarcations entre les régularités et les irrégularités ? Qu'est-ce qui dans un corpus vérifiera, exemplifiera et légitimera, dans la communication orale et écrite, la parole qui sera jugée naturelle, simple, utile, éventuellement belle, ou par contraste, décalée, parce que vieillie, fantaisiste, désordonnée, en échec, etc. ? Avec les aides documentaires, on procède à des investigations étendues, sur des textes diversifiés. Pour définir le français ordinaire contemporain, la prose littéraire est subtilement dosée avec les textes journalistiques, techniques, audiovisuels, les transcriptions d'entretiens, d'enquêtes, etc. L'exigence de précision dans les résultats reposera, si on veut la maintenir et la renforcer, sur une meilleure connaissance de ce qu'on extrait. 
Raffiner les analyses et les classifications passe par un réexamen des concordances fournies par la documentation automatique. Mais comment réalise-t-on une bonne documentation, notamment en lexique-syntaxe, et quels sont les bons concordanciers? La possibilité d'examiner des gros volumes textuels ne doit pas faire écran à la nécessité de parvenir à un dépassement des dosages qui se donnent à voir comme spontanés. Proches, par la méthode, de grammaires comme Le Bon Usage de Grevisse, ils relèvent du reproche d'« impressionnisme », qui fut fait en son temps à la grammaire traditionnelle par la grammaire générative et que l'analyse statistique, bien souvent trop sommaire en syntaxe, malgré d'indéniables succès sur d'autres terrains d'étude, ne suffit pas à corriger. Ce bilan débouche sur un examen critique de l'objetlangue.

\section{Les contributions}

Nous n'avons pas cherché à donner aux articles qui composent notre numéro l'apparence d'un ensemble trop cohérent, obéissant à une répartition contraignante des sujets traités. De notre point de vue, la notion de «semi-figement» apportait des observations fécondes si on évitait avec soin de les enfermer dans un statut de données « résiduelles » en face du figement et du non figement. Classée dans l'ordre alphabétique, chaque contribution pourra être lue indépendamment des autres. Complémentairement, on trouvera en fin de volume un résumé/abstract écrit par les auteurs présentant les commodités d'un index.

Onze études sur quatorze mettent l'accent sur la syntaxe du verbe et/ou de la préposition. D'où des recoupements nombreux sur les méthodes comme sur les données. On réfléchira d'autant mieux sur les choix opérés et sur leurs limites. Avec des redites comme la locution verbale casser sa pipe, c'est le phénomène de l'« exemple d'école» qui apparait et mérite réflexion. En contraste, on observera une diversification optimale de l'exemplification avec deux classifications, exploitables sur support électronique, qui prennent le lexique à l'échelle des lexicographes dans une visée d'exhaustivité. Celle de Françoise Dubois-Charlier, effectuée en collaboration avec Jean Dubois, comprend 7000 locutions du français. Celle de Jean Klein et Béatrice Lamiroy organise les expressions verbales figées, donc sensiblement les mêmes formes, dans une somme intégrant les variétés belges, québécoises et suisses. C'est une base de données (BFQS) de 45000 entrées. Ces points de vue très généraux sont complétés par la synthèse de Salah Mejri sur le figement absolu ou relatif et les degrés de figement à laquelle on ajoutera le bilan de Gerda Haßler et Christiane Hümmer sur figement et défigement polylexical.

Des segments de grammaire viennent enrichir ces survols généraux. Un des plus directement utiles est celui de Christian Molinier, à propos des constructions causatives en faire figées ou semi-figées (Léa fait bouillir la marmite, Paul a fait avaler la pilule à Léa, Luc s'est fait enguirlander). L'analyse syntaxique permet de classer un maximum d'emplois (150) à répertorier. Le classement est présenté dans une table de "lexique-grammaire», donnée intégralement en annexe. Cette contribution nous permet de visualiser, en détail et en taille réelle, un résultat typique des classifications précédemment citées. Toutes procèdent largement des travaux de Maurice Gross dans 
ce domaine. A propos des constructions verbales, Marie-Pierre Sales a étudié le déictique ça en position sujet. S'il existe bien un petit nombre de locutions (ça barde), en recoupement avec les exemples étudiés ici par Catherine Garitte (ça va ?), la plupart des expressions s'en distinguent par des propriétés compositionnelles: la présence du pronom conduit notamment à des rapprochements avec les constructions pronominales en se (ca craint). Un groupe de quatre articles porte sur la préposition et les continuums entre figement et non figement dans les constructions où elles entrent. Céline Vaguer a étudié la préposition dans à partir d'exemples de constructions verbales figées (pédaler dans la semoule); Badreddine Hamma et Yukiyo Homma, la préposition par dans les adverbiaux, génératrice de polylexicalité pour l'un (<entrer> par la petite porte, par hasard), source de l'approximation du lieu (par ici, par là, par là-bas), pour l'autre ; Belinda Lavieu, la préposition à introduisant un groupe prépositionnel (de moyen) (laver son linge à la main, à la machine).

L'article de Jean-Claude Anscombre traite du semi-figement et des stéréotypes à partir de la parole proverbiale. C'est une synthèse comparable à notre groupe d'articles initiaux. L'auteur rappelle que les possibilités de variation sont lexicales et syntaxiques, qu'elles ont lieu à l'intérieur de moules rythmiques contraignants. Pour en rendre compte, il propose la notion de «figement du deuxième type ». Une telle formulation permet de remettre à plat les typologies. En particulier, elle n'est pas incompatible avec une redéfinition des priorités. Et si, comme le proposent Jean Klein et Béatrice Lamiroy, le semi-figement était le problème central du figement? Un tel retournement permet notamment de renouer en syntaxe avec la prise en compte des séquences textuelles dont la consultation est optimisée par les aides documentaires. C'est en effet là qu'on puise habituellement les exemples de semi-figement dès lors qu'ils sont pensés en termes de défigement. Mais parler de défigement devient impropre si le semifigement précède le figement. Sur ce point précis, Gerda Haßler et Christiane Hümmer montrent à partir de cas diversifiés que la discussion est loin d'être épuisée si on refuse d'orienter a priori la relation entre figement et semi-figement dont la notion de degrés de figement, approfondie ici par Salah Mejri, est le point de départ obligé. Réinterpréter diverses figures rhétoriques et stylistiques et leurs associations offre des solutions multiples pour rendre compte des correspondances forme-sens. Antoinette Balibar-Mrabti réexamine la figure de l'allusion historique dans des mini-récits donnés par la tradition lexicographique des dictionnaires Larousse. Ils permettent d'étudier des parataxes, restreintes ici à un cas d'espèce (brûler ses vaisseaux). Comment dépasser les monographies lexicologiques? Quelle recherche documentaire aujourd'hui et pour quelle syntaxe?

Les articles de Catherine Garitte et de Bernard Laks abordent la syntaxe et le lexique à partir d'un autre centrage. Pour l'une, c'est l'acquisition du langage qui est l'intérêt premier. Elle s'appuie sur soixante et un gestes conventionnels répertoriés chez l'enfant et établit une comparaison avec des expressions idiomatiques. L'autre raisonne à partir de la phonologie. Dans une approche méthodologique de "grammaire de construction», il étudie la liaison comme test de cohésion et de figement syntaxique. Ces deux études entraient naturellement dans notre thématique d'ensemble et font apparaittre une dimension essentielle de la confrontation des contributions : l'interdisciplinarité de la plupart des discussions. Nous en avons signalé 
un exemple à propos des déictiques en rapport avec les gestes. On pourrait également juxtaposer « métrique naturelle » dans les proverbes et liaison.

\section{RÉFÉRÉRENCES}

Auroux, S. (1994), La révolution technologique de la grammatisation, Liège, Pierre Mardaga.

BANNIARD, M. (2003), «Latinophones, romanophones, germanophones : interactions identitaires et construction langagière (VIIIe - IXe siècle) », Médiévales, 45.

Chevalier, J.-C. (2006), Histoire de la syntaxe. Naissance de la notion de complément dans la grammaire française (1530-1750), Paris, Honoré Champion (1ère éd. 1968).

Chevalier, Y. \& Wahl, P., éds. (2006), La syllepse. Figure stylistique, P.U.L.

François, J., LeEman, D. \& LE Pesant D., éds. (2007), (mars), Langue française, 153 : Autour du dictionnaire des verbes de J. Dubois et F. Dubois-Charlier.

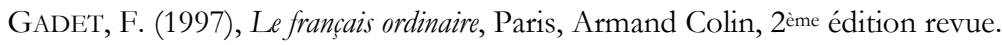

GonZALEZ-ReY, I. (2001), La phraséologie du français, Mirail-Toulouse, Presses Universitaires.

Gross, G. (1996), Les expressions figées en français, noms composés et autres locutions, Paris, Ophrys.

Gross, M. (1976), «Présentation » in BoOns, J.-P., Guillet, A., LeClÈre, C., éds., La structure des phrases simples en français, constructions intransitives, Genève, Droz.

Gross, M. (1982), «Une classification des phrases «figées» du français », Revue Québécoise de Linguistique, $11: 2$.

HABERT, B. (2005), Instruments et ressources électroniques pour le français, Paris, Ophrys.

KLEIBER, G. (2003), « Faut-il dire adieu à la phrase ? », L'Information grammaticale, 98.

LEEMAN, D. éd. (2003), Linx, 48, Approches syntaxiques contemporaines.

Maingueneau, D. (2002), "Linguistique et littérature: le tournant discursif », Vox poetica, www.vox-poetica.net.

Martins-BAltar, M. éd. (1997), La locution entre langue et usage, ENS EDITIONS, Fontenay/Saint-Cloud, Paris, Ophrys.

Mel'ČUK, I. A., Clas, A., POlguÈRe, A. (1995), Introduction à la lexicologie explicative et combinatoire, Louvain-la-Neuve, Duculot.

Meschonnic, H. (1991), Des mots et des mondes, dictionnaires, encyclopédies, grammaires, nomenclatures, Paris, Hatier.

Rossellini, M. (1999), «Les mots sans guère de choses : la praelectio », Langue française, 121.

Ruwet, N. (1964), «La linguistique générale aujourd'hui », Archives européennes de sociologie, EPHE, V, vol. 2.

Ruwet, N. (1983), «Du bon usage des expressions idiomatiques dans l'argumentation en syntaxe générative », Recherches linguistiques, 11. 
